\title{
ARTICLE
}

\section{In situ observation of step-edge in-plane growth of graphene in a STEM}

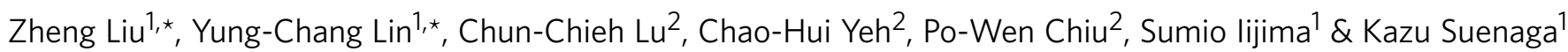

It is extremely difficult to control the growth orientation of the graphene layer in comparison to Si or III-V semiconductors. Here we report a direct observation of graphene growth and domain boundary formation in a scanning transmission electron microscope, with residual hydrocarbon in the microscope chamber being used as the carbon source for in-plane graphene growth at the step-edge of bilayer graphene substrate. We show that the orientation of the growth is strongly influenced by the step-edge structure and areas grown from a reconstructed 5-7 edge are rotated by $30^{\circ}$ with respect to the mother layer. Furthermore, single heteroatoms like Si may act as catalytic active sites for the step-edge growth. The findings provide an insight into the mechanism of graphene growth and defect reconstruction that can be used to tailor carbon nanostructures with desired properties.

\footnotetext{
${ }^{1}$ Nanotube Research Center, National Institute of Advanced Industrial Science and Technology (AIST), Tsukuba 305-8565, Japan. ${ }^{2}$ Department of Electrical Engineering, National Tsing Hua University, Hsinchu 30013, Taiwan. * These authors contributed equally to this work. Correspondence and requests for materials should be addressed to Z.L. (email: liu-z@aist.go.jp).
} 
S ince the first demonstration of producing graphene layers by mechanical exfoliation of bulk graphite ${ }^{1}$, many growth or synthesis approaches for producing few-layer graphene have been investigated, including chemical vapour deposition (CVD) on metal catalysts ${ }^{2-8}$, reduced graphene oxides ${ }^{9}$, thermal decomposition of $\mathrm{SiC}^{10,11}$ and molecular beam epitaxy ${ }^{12-14}$. However, in comparison with Si or III-V semiconductors, it is extremely difficult to control the growth orientation of the graphene layer, and hence its assembly in an electronic device is believed to be problematic. Direct visualization of how the graphene network evolves during growth is highly desired in an effort to precisely understand the atomic processes of the growth mechanism. Furthermore, to realize well-controlled graphene nanodevices, identifying the orientation relationship between the seed crystal and the growing layer or detecting the influence of the catalytic atoms during growth would be of immense benefit. The extremely high CVD growth rate (on the order of micrometres per minute) makes atomic resolution analysis during this method unfeasible.

The edge structures of graphene ribbons are of fundamental importance during growth and preparation processes because the electronic, optical, magnetic, mechanical and electrochemical properties depend not only on the ribbon width but also on the edge structures ${ }^{15-25}$. Graphene nanoribbons generally have edge structures that are a combination of armchair (AC) and zigzag (ZZ) geometries ${ }^{26,27}$, and recent theoretical calculations and experimental studies have also demonstrated the existence of an alternate pentagon-heptagon edge structure ( $\mathrm{ZZ} \mathrm{(57))}$ reconstructed from a periodic hexagon $Z Z$ structure ${ }^{20,28-33}$. Graphene nanoribbons with $\mathrm{AC}$ and $\mathrm{ZZ}$ edges have distinct electronic transport properties ${ }^{34}$, but atomic-scale edge defects can significantly affect the electronic properties ${ }^{35}$. Pristine graphene edge structures are of particular importance as they govern the quality of the subsequent synthesized material. Lowenergy electron microscopy, photoemission electron microscopy and low-energy electron diffraction can give unique insight into the fundamental growth mechanisms of graphene on metal substrates $^{36,37}$. Invaluable insight into the atomic structure of graphene including defects and edge stabilities has been provided by scanning tunnelling microscopy ${ }^{38,39}$ and transmission electron microscopy (TEM), especially aberration-corrected highresolution $\mathrm{TEM}^{33,40-48}$, and furthermore, the electron beam can be used to modify the graphene edge structure, create single atomic C chains or nanoribbons ${ }^{49,50}$, sculpt near-defect-free graphene nanostructures ${ }^{48}$, sublimate graphene layer by layer ${ }^{51}$ and reknit holes in monolayer graphene ${ }^{52}$.

As the vacuum system in an electron microscope is not perfect, the residual hydrocarbon gas in the sample chamber can be used to grow graphene at high temperatures, although the properties of the hydrocarbon gases cannot be characterized. Here we show that graphene grows extremely slowly (several angstroms per minute) in a transmission electron microscope, allowing simultaneous growth and atomic resolution imaging. We visualize the in situ in-plane growth of graphene on the terrace of the first layer using aberration-corrected scanning TEM (STEM), and observe the activity of a single $\mathrm{Si}$ atom catalyst during graphene growth on an atomic scale. STEM has the added advantage in that the focused high-density electron beam is able to modify the structure of the sample, and thus, this method can be used to manipulate the graphene growth. Although Zan et al. ${ }^{52}$ have reported reknitting holes in monolayer graphene with the reknitted areas that are composed of many 5-7 defects showing an 'amorphous' characteristic, the mechanism of in-plane growth from a step-edge in this study is different from that of reknitting holes. Holes have a limited circumference without substrate, however, edges have a half opened space to grow graphene. More importantly, the newly grown graphene is not amorphous but crystalline in our study. The relationship between the growth speed of graphene and the residual hydrocarbon gas pressure is also investigated.

\section{Results}

Step-edge graphene growth. Figure la shows a scanning electron microscopy image of a single-crystal bilayer graphene (SC-BLG) grown using ambient pressure $\mathrm{CVD}^{53}$. Both the first
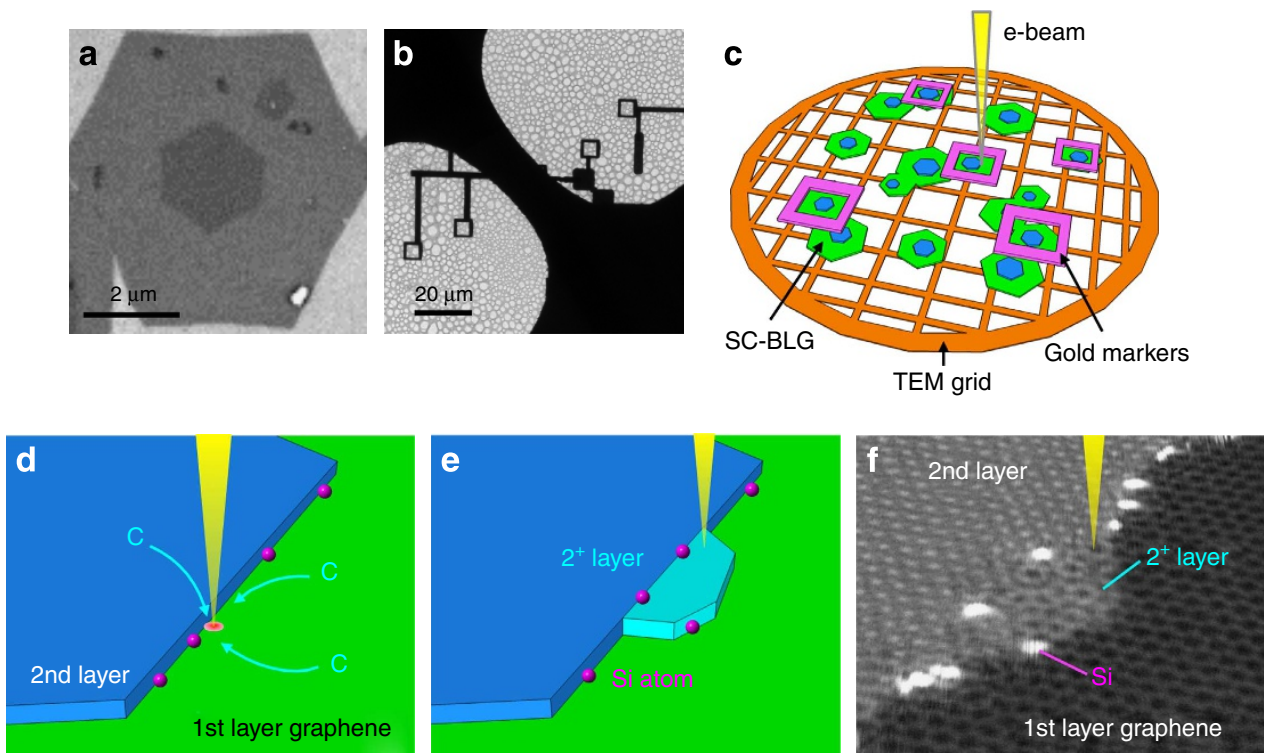

Figure 1 | Scheme of graphene growth by e-beam in TEM. (a) Scanning electron microscopy image of a SC-BLG, (b) marked by e-beam lithography, (c) transferred to a Mo TEM grid and (d) schematic drawing of the 2nd-layer graphene step-edge. Hydrocarbon accumulates to the e-beam scanning area. (e) The new growth graphene layer ( $2^{+}$layer, light blue) at the BLG step-edge. Single Si atoms (pink) located at the step-edge. (f) ADF image in a perspective view (the ADF image is $40^{\circ}$ inclined respect to the vertical plane, the raw image is shown in Supplementary Fig. 3) showing the electron-beam-induced growth from the step-edge of the SC-BLG where the single Si atoms (brighter contrast) existed. 
and second graphene layers exhibit hexagonal shape and the macroscopically sharp edges serve as an ideal step structure for the assembly of foreign impurities. SC-BLG flakes with distinct twisting angles were marked by electron-beam (e-beam) lithography and then cleanly transferred to a Mo TEM grid as shown in Fig. $1 \mathrm{~b}$ (ref. 53). The marked SC-BLG flakes were observed at various temperatures $\left(T=\mathrm{RT}\right.$ to $\left.700^{\circ} \mathrm{C}\right)$ in an aberrationcorrected STEM instrument operated at $60 \mathrm{kV}$ (Fig. 1c).

Carbon materials placed in the electron beam usually suffer from radiation damage, and Kotakoski et al. ${ }^{32}$ found that to prevent knock-on damage in graphene the electron energy should be below a threshold of $\sim 50 \mathrm{kV}$, even for defective samples and irradiation at the edges. Knock-on damage is thus likely to be observed even at the edges of graphene under a $60-\mathrm{kV}$ irradiation, but because of the imperfect vacuum $\left(1-3 \times 10^{-5} \mathrm{~Pa}\right.$ around the specimen), graphene growth (the completely opposite phenomenon to knock-on damage) can be observed at a particular temperature when the graphene samples are heated inside the electron microscope. The nature of the residual gases inside the microscope chamber cannot be identified, but it is well known that they are a mixture of hydrocarbon gases. Hydrocarbons will agglomerate in the electron-beam scanning area and graphitize from the bilayer edge. Figure 1d,e shows the schematics of the graphene growth, and Fig. If shows an annular dark-field (ADF) image of the electron-beam-induced growth from the step-edge of the SC-BLG in a perspective view. At room temperature, the electron beam will usually result in contamination of the graphene surface, whereas at temperatures higher than $700^{\circ} \mathrm{C}$, growth of graphene is never found due to the high mobility of the hydrocarbon atoms.

Figure 2 shows a series of ADF images of a bilayer singledomain graphene heated to $500^{\circ} \mathrm{C}$ inside the microscope, revealing the growth process from the edge of the twisted bilayer graphene. To explain these results in a clear manner, we label the graphene layer with the larger planar dimension as layer 1 (or 1st) and that with the smaller area as layer 2 (or 2nd). Note, however,
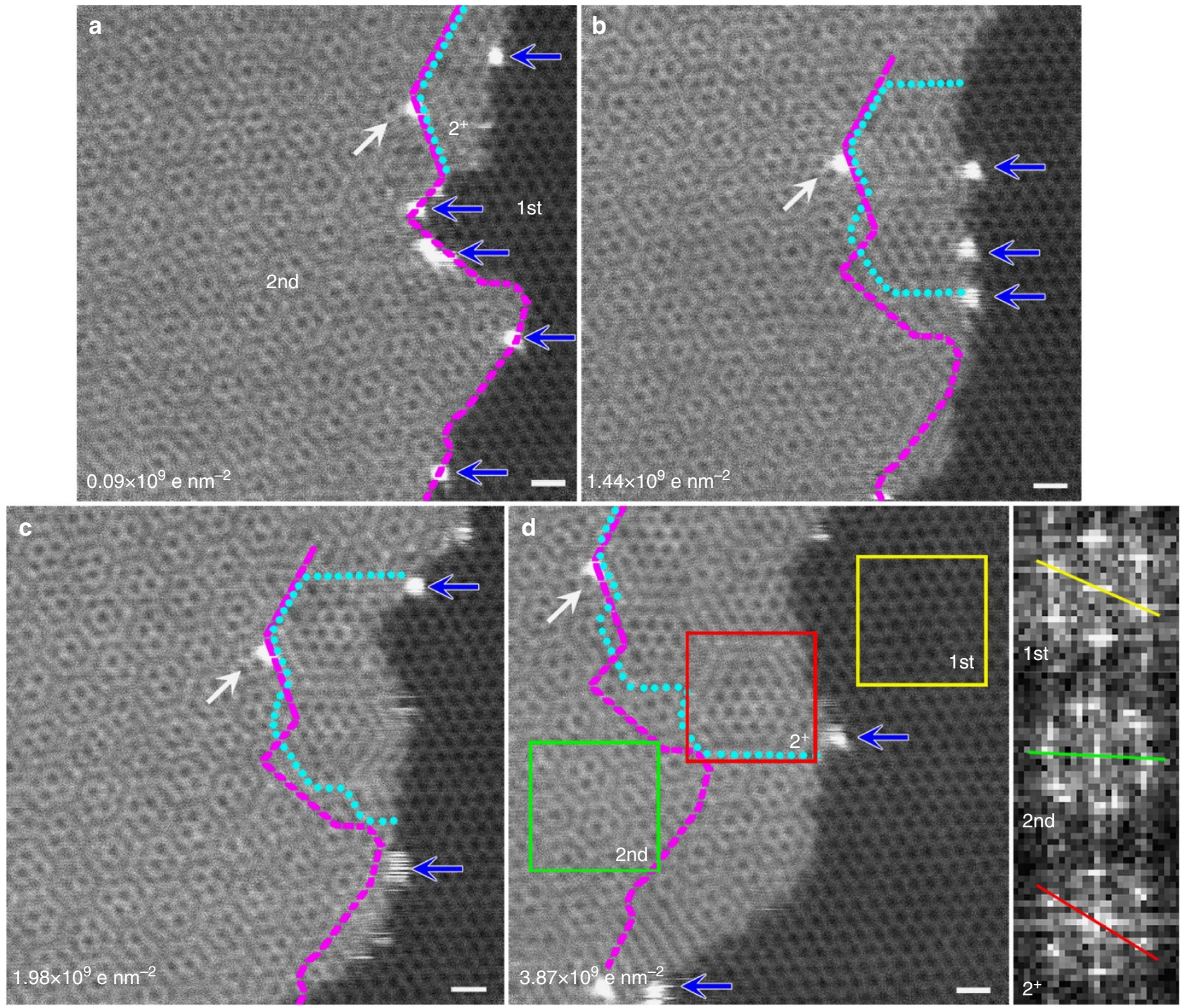

Figure 2 | Sequential ADF images showing graphene from the step-edge of the BLG. The initial 2nd-layer step-edge is indicated by dashed magenta line, while the rotated $2^{+}$-layer highlighted by cyan dot line shows different moiré patterns and increases over time. (a) Initial state (beam density is $0.09 \times 10^{9} \mathrm{enm}^{-2}$ ), (b) after beam scanning with a cumulative electron dose of $1.44 \times 10^{9} \mathrm{enm}^{-2}$, (c) after beam scanning with a cumulative electron dose of $1.98 \times 10^{9} \mathrm{enm}^{-2}$ and (d) after beam scanning with a cumulative electron dose of $3.87 \times 10^{9} \mathrm{enm}^{-2}$. The inset shows the Fourier transform images corresponding to the 1st (yellow rectangle), 2nd (green rectangle) and $2^{+}$(red rectangle) layer of graphene. Blue arrows show the single Si atoms at step-edge and white arrow indicates the pinned Si atom. The sample is heated to $500^{\circ} \mathrm{C}$ in TEM chamber. Scale bar, $0.5 \mathrm{~nm}$. 

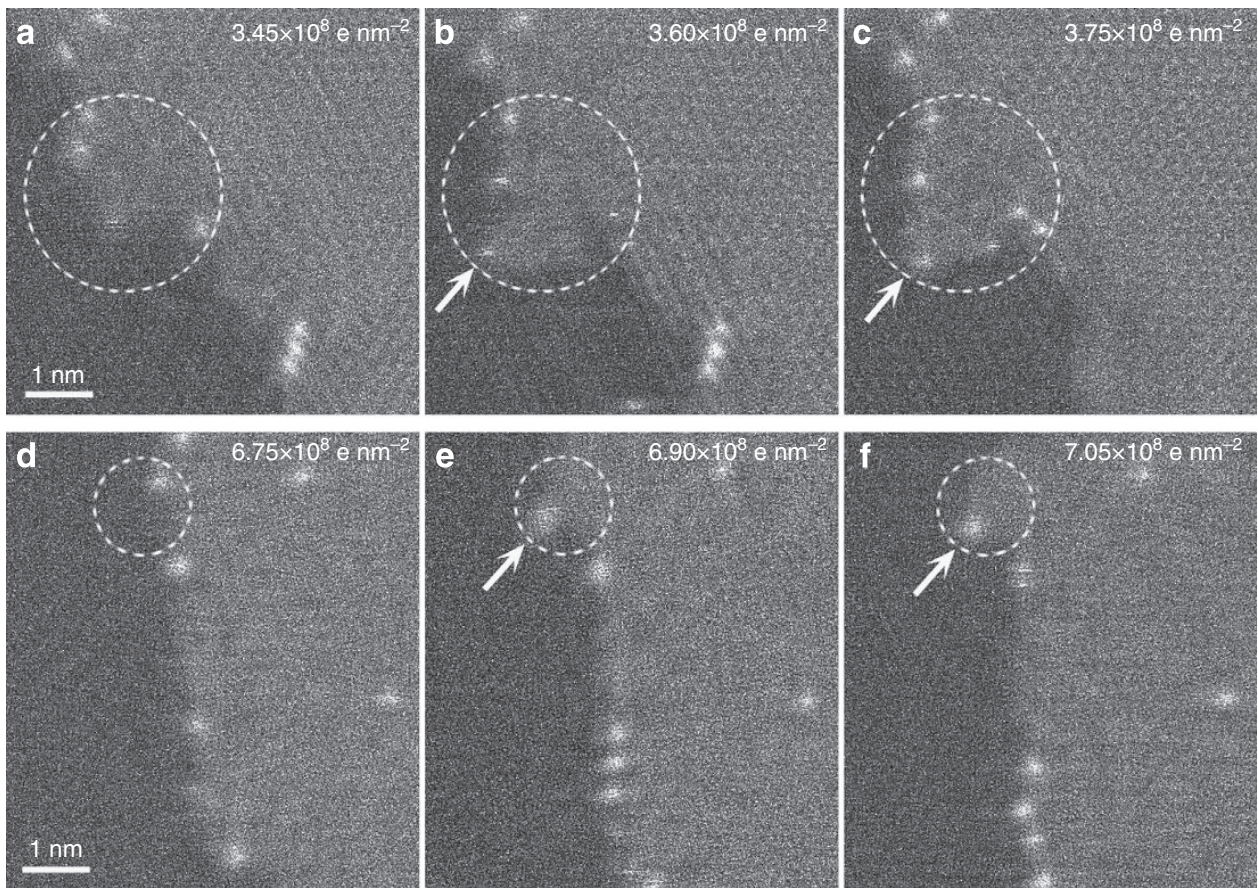

Figure 3 | Si atoms act as catalysts during graphene growth from BLG step-edge. Some highlighted continuous frames in Supplementary Movie 2, (a-c) and in Supplementary Movie 3, (d-f). The white arrows indicate Si atoms. By comparing the areas inside the dashed circles, new growth of graphene can be clearly seen. The images are taken at $650^{\circ} \mathrm{C}$ at a higher frame rate. The cumulative electron doses during beam scanning are: (a) $3.45 \times 10^{8} \mathrm{enm}^{-2}$, (b) $3.60 \times 10^{8} \mathrm{enm}^{-2}$, (c) $3.75 \times 10^{8} \mathrm{enm}^{-2}$, (d) $6.75 \times 10^{8} \mathrm{enm}^{-2}$, (e) $6.90 \times 10^{8} \mathrm{e} \mathrm{nm}^{-2}$ and (f) $7.05 \times 10^{8} \mathrm{e} \mathrm{nm}-2$.

that this does not clarify which layer is on the top. In the corresponding Fourier transforms of the images (Fig. 2 inset), we see that the twisting angle between the two layers is about $20^{\circ}$. The dashed blue line in Fig. 2a indicates the new growth area at the beginning of the growing stage, and it is worth noting here that there are some isolated brighter atoms in this area. According to an analysis of the electron energy-loss spectroscopy (EELS) data (Supplementary Fig. 1), these brighter atoms are Si atoms introduced by the quartz furnace used during CVD. These Si atoms might act as catalysts during the graphene growth and slide over the graphene edges during electron-beam scanning. The possibility of catalytic effect of $\mathrm{Si}$ atoms can be demonstrated from the movies and several highlighted frames shown in Fig. 3 and Supplementary Movies 1-3. Graphene usually grows where there exist $\mathrm{Si}$ atoms and the $\mathrm{Si}$ atoms are pushed to the front growth edge forming a local protrusion on the growth area behind the Si atom indicated by the circles and arrows in Fig. 3. Such catalytic effect of $\mathrm{Si}$ is similar to the tip-growth mechanism in carbon nanotube growth, while the metallic catalyst particles are always pushed to the growth tip of carbon nanotubes. Unless the $\mathrm{Si}$ atoms are pinned by defects, they are usually pushed to the outermost graphene edges by further growth, as indicated by the blue arrows in Fig. 2. The pinned Si atom, shown by the white arrow in Fig. 2a, gives the reference position of the pristine edge and acts as a marker to compare new growth areas. Over time, the gradually growing areas in the 2nd layer (enclosed by the dotted cyan lines in Fig. 2b-d) can be clearly identified from the pristine areas by the different moiré patterns. This demonstrates that new growth from the edge of the 2 nd layer occurs when the sample is heated to $500{ }^{\circ} \mathrm{C}$ inside the electron microscope. From the contrast of the ADF images, we can see that the new growth area (labelled by $2^{+}$in Fig. 2) is in the same plane as the 2 nd layer (that is, in-plane growth) but some areas have a different planar direction. The $2^{+}$area includes both unrotated and rotated areas, and because there is no clear boundary between the unrotated $2^{+}$ and the original 2nd-layer areas, it is more difficult to identify the unrotated area. If two continuous images with a common reference point, in this case, the pinned $\mathrm{Si}$ atom, are compared, then the new growth area can be identified directly: the areas to the right of the dashed magenta lines in Fig. 2 are new growth areas. A whole comparison of the new growth areas for each pair of images is shown in Supplementary Fig. 2. The angle between the rotated $2^{+}$area and the 1 st layer can be obtained by comparing the respective Fourier transform images (that is, those labelled $2^{+}$and 1st, respectively, in the inset), and we obtained a rotation angle of about $10^{\circ}$, indicating that the angle between the pristine 2 nd-layer area and the rotated further growth areas in the 2nd layer is about $30^{\circ}$.

Growth mechanisms determined by step-edge structure. The existence of pentagon-heptagon pairs between the 2 nd and the $2^{+}$areas causes such rotation $\left(30^{\circ}\right)$ and can be clearly seen in Fig. 4. Figure $4 \mathrm{a}$ is a filtered image of Fig. $2 \mathrm{~d}$ in which only the $\mathrm{C}$ atoms in layer 2 are shown (reflections corresponding to atoms in the 1st layer were deleted from a Fourier transform of Fig. 2d, and an inverse Fourier transform of the remaining spots gives the contrast of the $\mathrm{C}$ atoms in layer 2). The dashed magenta line separates the pristine area in the 2 nd layer (left-hand side) from the new growth in the $2^{+}$areas (right-hand side). The atomic growth processes can be identified from the image in Fig. $4 \mathrm{~b}$. The growth mechanisms can be classified into four categories: (i) from $\mathrm{ZZ}$ to $\mathrm{ZZ}$ (Fig. 4c), (ii) from reconstructed $\mathrm{ZZ} \mathrm{(57)} \mathrm{to} \mathrm{AC}$ (Fig. 4d), (iii) from AC to AC (Fig. 4e) and (iv) from reconstructed AC (57) to ZZ (Fig. 4f). The step-edges in the original area (magenta lines in Fig. $4 \mathrm{c}-\mathrm{f}$ ) act as seeds for the new growth. The three growth mechanisms (i), (iii) and (iv) are found in Fig. $4 \mathrm{~b}$. The new growth area can be divided into two domains: $2^{+} \mathrm{A}$ and $2^{+} \mathrm{B}$. The $2^{+} \mathrm{B}$ domain, which has the same orientation as the original 2nd layer, is an area grown from $\mathrm{ZZ}$ and $\mathrm{AC}$ 

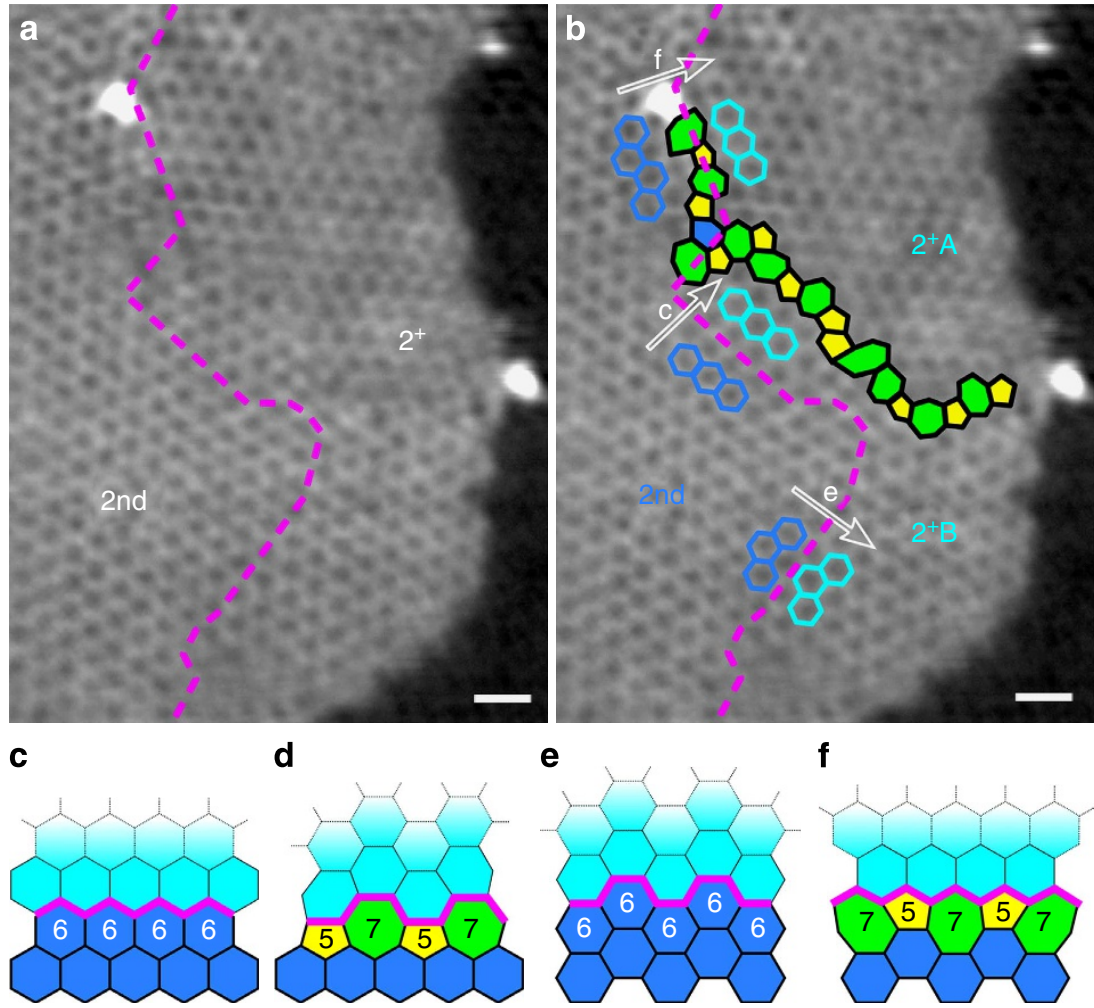

e

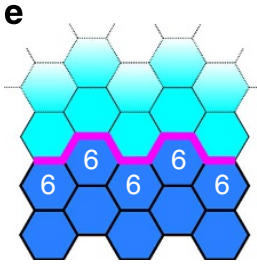

f

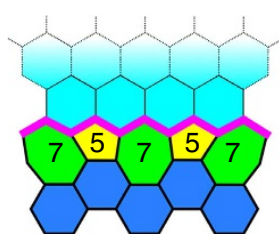

Figure 4 | Growth model determined by step-edge structure. (a) A filtered image of Fig. $2 \mathrm{~d}$ only showing the 2 nd and $2^{+}-$layer graphene. The dashed magenta lines indicate the edge of initial 2nd layer. (b) Identical image to a with highlighted 5-7 defect structure (yellow pentagon and green heptagon). Blue hexagons stand for the initial 2nd-layer structure, from where the $2^{+}$layer (cyan hexagons) grows along the white arrows. (c-f) Four categories of the growth model. (c) From ZZ to ZZ, (d) from reconstructed $Z Z$ (57) to $A C$, (e) from $A C$ to $A C$ and (f) from reconstructed AC (57) to ZZ. Scale bar, $0.5 \mathrm{~nm}$.

edges, while the $2^{+} \mathrm{A}$ domain, which is rotated by $30^{\circ}$ with respect to the original 2nd layer, is grown from a reconstructed AC (57) edge. At the boundary of the two domains, pentagonheptagon pairs appear, as shown in Fig. $4 \mathrm{~b}$. The formation of such a grain boundary is similar to that formed in polycrystalline graphene synthesized by CVD.

The same phenomenon of $30^{\circ}$ rotating growth from the $\mathrm{AB}$ stacking bilayer graphene edge is shown in Supplementary Fig. 3. The new growth $2^{+}$area indicated by the cyan rectangle has a different moire pattern from that of the original 2nd layer. By applying the same method used for Fig. 4a, we again found that the zigzag chains of the $2^{+}$and 2 nd-layer areas had a rotation angle of about $30^{\circ}$. ADF images of bilayer graphene with a nearly $30^{\circ}$ twisting angle between layers 1 and 2 are shown in Supplementary Fig. 4, and those images also show that there is an almost $30^{\circ}$ rotation of the zigzag chains between the $2^{+}$and original 2 nd-layer areas. This $30^{\circ}$ rotation is due to the $5-7$ pairs on the 2nd-layer edge before growth. These 5-7-pair-terminated edges can be found in monolayer graphene or some bilayer graphene as shown in Supplementary Fig. 5. Zigzag edges are metastable $e^{20}$, and a planar reconstruction of the zigzag edge into the recently reported $\mathrm{ZZ} \mathrm{(57)} \mathrm{edge} \mathrm{structure} \mathrm{was} \mathrm{noted} \mathrm{by}$ Koskinen et al. ${ }^{20}$ to lead to a lower edge energy and a selfpassivating edge that would not bond with hydrogen atoms. This is the first time that the 5-7 structure has been found on the edge of the 2nd layer of bilayer graphene, from which new growth is rotated by $30^{\circ}$ with respect to the original layer. Although $\mathrm{AB}$ stacking is the most preferable stacking sequence in graphite, in the case of few-layer (at least two) graphene, it would seem that the growth is determined by the edge structure, as shown in Fig. $4 \mathrm{c}-\mathrm{f}$.
Such growth is only found in the 2 nd layer (the 1st layer is a suspended layer), which means that a substrate is necessary for in-plane graphene growth. Another interesting result is that the two open edges of the 1st and 2nd layers will form a closed edge structure once the separation between the two is below a critical distance. This can be seen in Supplementary Fig. 6 and Supplementary Movie 4.

Step-edge graphene growth rates. We can obtain a rough estimate of the growth rate by measuring the change in the size of the growth areas from series of sequential ADF images. The number of additional $\mathrm{C}$ atoms can be determined by counting the bright spots that represent $\mathrm{C}$ atoms in the ADF images or deduced from the increase in the size of the area; these two methods are equivalent within their respective errors. Figure 5a shows the temperature range in which the crystalline graphene grows in our study $\left(500^{\circ} \mathrm{C}<T<700^{\circ} \mathrm{C}\right)$. At $T<500{ }^{\circ} \mathrm{C}$, the graphene does not grow in a crystalline structure but only amorphous carbon agglomerations are found on the first and second layer. On the other hand, the graphene edge is being etched during the observation at $T>700^{\circ} \mathrm{C}$. This growth condition of graphene under the electron beam is different from the typical CVD conditions. To perceive the growth rate dependence of the residual hydrocarbon gas pressure, we have attempted experiments with different vacuum conditions. Figure $5 b$ shows the growth rates at three different residual hydrocarbon gas pressures. The pressure range is limited to $1.0-2.9 \times 10^{-5} \mathrm{~Pa}$ for keeping the TEM workable condition. The growth rate seems to be affected by the residual gas pressure. There is a tendency that if there are more residual hydrocarbon gases left in the TEM chamber, the growth rate will be faster. 

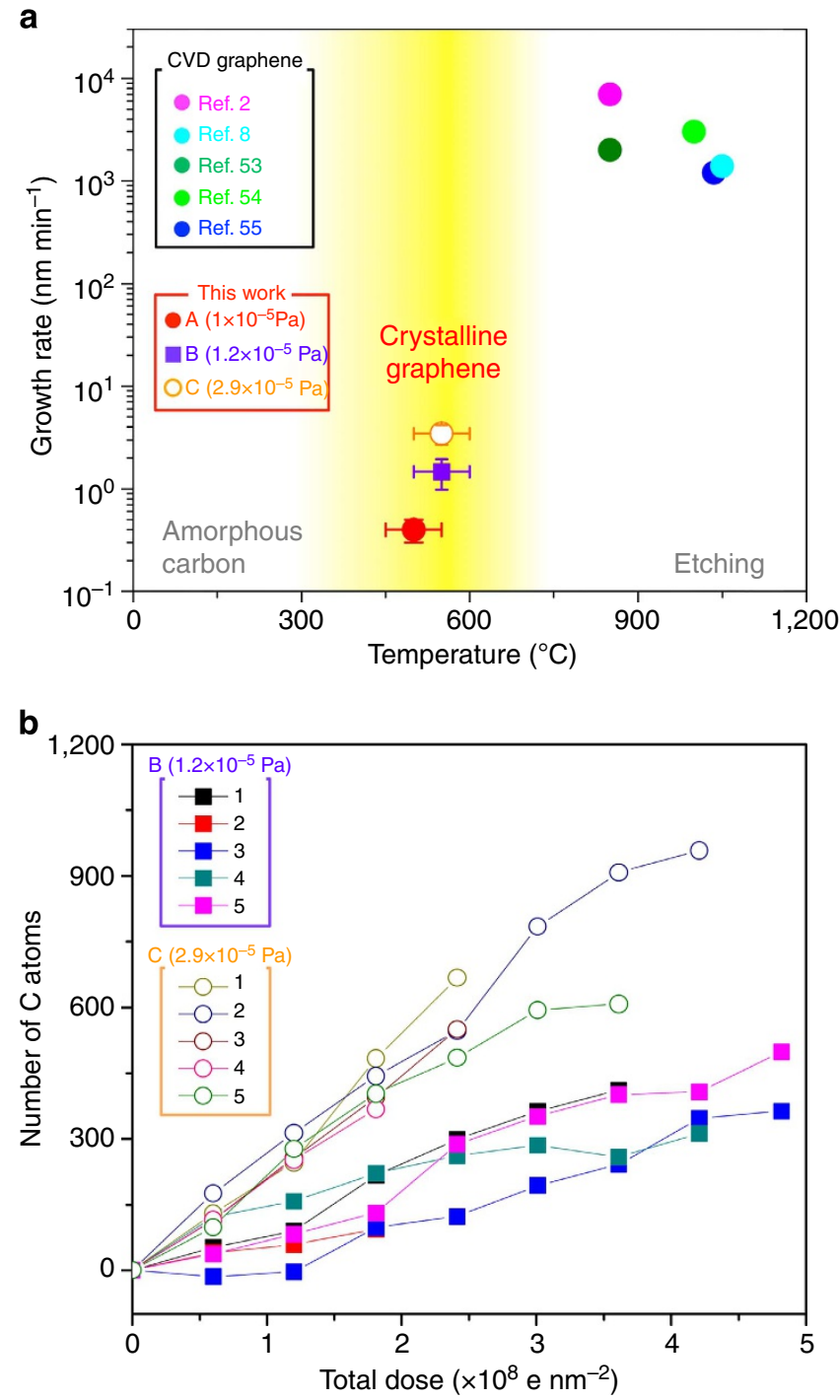

Figure 5 | Step-edge growth rate. (a) Comparison between high-speed growth (CVD) and low-speed growth in this study. In this study, between 500 and $700{ }^{\circ} \mathrm{C}$, graphene grows from step-edge. Three different growth rates at different residual hydrocarbon gas pressures are shown. The growth rates at $2.9 \times 10^{-5} \mathrm{~Pa}$ are shown by circles and the growth rates at $1.2 \times 10^{-5} \mathrm{~Pa}$ are shown by rectangles. When $T<500^{\circ} \mathrm{C}$, amorphous carbons appear as contaminations. If $T>700^{\circ} \mathrm{C}$, carbon atoms at stepedges are etched and no more graphene growth is found. (b) The growth speed measurements on different selected areas repeated five times at each residual hydrocarbon gas pressure for $1.2 \times 10^{-5}$ and $2.9 \times 10^{-5} \mathrm{~Pa}$.

We know that the step-edge in-plane growth seen here is not just simple physical absorption of $\mathrm{C}$ atoms because single $\mathrm{Si}$ catalyst atoms are present at the bilayer edges. The electron beam also plays an important role in the growth by accumulating hydrocarbon gas at the scanned area; there is no growth in areas far from the scanned area as shown in Supplementary Fig. 7. It is well known that graphene growth is strongly dependent on the number of available $\mathrm{C}$ atoms that are able to bond within a certain time, that is, if a large number of $\mathrm{C}$ atoms encounter graphene edges and do not have enough time to locate an energetically optimal location before forming covalent bonds, then the graphene will just be contaminated with amorphous C. This is the case when graphene is observed in the STEM at RT and low-vacuum conditions. However, if the substrate graphene is heated to $500-700{ }^{\circ} \mathrm{C}$, then the incident $\mathrm{C}$ atoms are more likely to form $\mathrm{sp}^{2}$ bonds with the step-edge atoms, and in-plane graphene growth will occur. At temperatures above $700^{\circ} \mathrm{C}$, the etching effect is dominant, and no growth was observed (Fig. 5). As shown in Fig. 5, the growth rates found in this study were very low (several angstroms per minute) in comparison with that of $\mathrm{CVD}^{53-55}$ (several micrometres per minute). Even though the vacuum conditions and gas source pressure were different from those in CVD, we were still able to successfully induce graphene growth and observe the growth at atomic resolutions.

Such in situ growth could be used to precisely design the orientation of graphene or other heterobilayer materials. When the pristine seed edges are terminated with $\mathrm{ZZ}$ or AC edges, the orientation of new growth area will remain the same; when the seed edges are terminated with some 5-7 defects, the new growth area will rotate by $30^{\circ}$ with respect to the original layer. If the seed edges can be modified to terminate with other kinds of defects, rotation angle between $0^{\circ}$ and $30^{\circ}$ will be achieved. Since the growth speeds are dependent on the residual hydrocarbon gas pressures, observations by using environmental electron microscope with controlled carbon source are expected to offer more important information and attract attention to techniques controlling the growth of graphene or similar heterobilayer materials.

\section{Methods}

Graphene synthesis and transfer. SC-BLG were grown by the ambient pressure CVD of methane (99.99\%) on polycrystalline $\mathrm{Cu}$ foil. Before growth, the $\mathrm{Cu}$ foil was soaked in acetic acid for $30 \mathrm{~min}$ to remove the surface oxides. The $\mathrm{Cu}$ foil was then mounted in the CVD chamber and the furnace was ramped up to $1,050^{\circ} \mathrm{C}$ over $40 \mathrm{~min}$, with constant flow of $\mathrm{Ar}$ ( 300 s.c.c.m.) and $\mathrm{H}_{2}$ (10 s.c.c.m.). After reaching $1,050^{\circ} \mathrm{C}$, the sample was annealed for $90 \mathrm{~min}$ without changing the gas flow. For graphene growth, methane (80 p.p.m.) was mixed with the flows of $\mathrm{Ar}$ (300 s.c.c.m.) and $\mathrm{H}_{2}$ (15 s.c.c.m.). $\mathrm{H}_{2}$ was fed into the reaction chamber for $\sim 7$ min to form bilayer graphene. After the growth, the $\mathrm{Cu}$ foil was moved to the cooling zone.

For the transfer process, the as-grown graphene sheet on $\mathrm{Cu}$ foil was first spin coated with a layer of polycarbonate, followed by etching in $\mathrm{HCl}$ aqueous solution to remove the $\mathrm{Cu}$ foil. The polycarbonate film and the attached graphene flakes were then transferred onto a Mo-quantifoil grid for TEM observations. EDS analyses demonstrate that there is no Cu left.

STEM and EELS observations. ADF imaging and EELS analysis were performed on a JEOL $2100 \mathrm{~F}$ with a cold field-emission gun and an aberration corrector (the DELTA-corrector) operating at $60 \mathrm{kV}$. A Gatan GIF Quantum was used for the EELS chemical analyses. Typically, a $0.11-\mathrm{nm}$ resolution was achieved for the STEM ADF imaging. The inner and outer collection angles for the ADF image ( $\beta_{1}$ and $\beta_{2}$ ) were 58 and $129-140 \mathrm{mrad}$, respectively. The beam current was about $15 \mathrm{pA}$ for the ADF imaging and EELS chemical analyses. The images shown in this paper are taken at high temperature (from 500 to $650{ }^{\circ} \mathrm{C}$ ), while the experiments have been performed at a wider range of temperatures (from 300 to $1,000 \mathrm{~K}$ ). Scan rate is $38 \mu$ s per pixel for faster scan and $58 \mu$ s for atomic resolution image. The images are filtered by a commercial software named HREM-Filters Pro. Vacuum conditions for different residual gas pressures were obtained by switching the trap pump on/off and by controlling the evacuation time.

\section{References}

1. Novoselov, K. S. et al. Electric field effect in atomically thin carbon films. Science 30, 666-669 (2004).

2. Sutter, P. W., Flege, J.-I. \& Sutter, E. A. Epitaxial graphene on ruthenium. Nat. Mater. 7, 406-411 (2008).

3. Kim, K. S. et al. Large-scale pattern growth of graphene films for stretchable transparent electrodes. Nature 457, 706-710 (2009).

4. Reina, A. et al. Large area, few-layer graphene films on arbitrary substrates by chemical vapor deposition. Nano Lett. 9, 30-35 (2009).

5. Li, X. et al. Large-area synthesis of high-quality and uniform graphene films on copper foils. Science 324, 1312-1314 (2009).

6. Lu, C.-C. et al. Characterization of graphene grown on bulk and thin film nickel. Langmuir 27, 13748-13753 (2011).

7. Robertson, A. W. \& Warner, J. H. Hexagonal single crystal domains of few-layer graphene on copper foils. Nano Lett. 11, 1182-1189 (2011).

8. Lu, C.-C. et al. Twisting bilayer graphene superlattices. ACS Nano 7, 2587-2594 (2013). 
9. Eda, G., Fanchini, G. \& Chhowalla, M. Large-area ultrathin films of reduced graphene oxide as a transparent and flexible electronic material. Nat. Nanotechnol. 3, 270-274 (2008).

10. Berger, C. et al. Electronic confinement and coherence in patterned epitaxial graphene. Science 312, 1191-1196 (2006).

11. Emtsev, K. V. et al. Towards wafer-size graphene layers by atmospheric pressure graphitization of silicon carbide. Nat. Mater. 8, 203-207 (2009).

12. Park, J. et al. Epitaxial graphene growth by carbon molecular beam epitaxy (CMBE). Adv. Mater. 22, 4140-4145 (2010)

13. Maeda, F. \& Hibino, H. Molecular beam epitaxial growth of graphene using cracked ethylene -advantage over ethanol in growth. Diamond Relat. Mater. 34, 84-88 (2013).

14. Maeda, F. \& Hibino, H. Growth of few-layer graphene by gas-source molecular beam epitaxy using cracked ethanol. Phys. Status Solidi B 247, 916-920 (2010).

15. Seitsonen, A. P., Saitta, A. M., Wassmann, T., Lazzeri, M. \& Mauri, F. Structure and stability of graphene nanoribbons in oxygen, carbon dioxide, water, and ammonia. Phys. Rev. B 82, 115425 (2010).

16. Ritter, K. A. \& Lyding, J. W. The influence of edge structure on the electronic properties of graphene quantum dots and nanoribbons. Nat. Mater. 8, 235-242 (2009).

17. Singh, A. K. \& Yakobson, B. I. Electronics and magnetism of patterned graphene nanoroads. Nano Lett. 9, 1540-1543 (2009).

18. Bhowmick, S. \& Shenoy, V. B. Edge state magnetism of single layer graphene nanostructures. J. Chem. Phys. 128, 244717 (2008).

19. Ponomarenko, L. A. et al. Chaotic Dirac billiard in graphene quantum dots. Science 320, 356-358 (2008).

20. Koskinen, P., Malola, S. \& Häkkinen, H. Self-passivating edge reconstructions of graphene. Phys. Rev. Lett. 101, 115502 (2008).

21. Yang, L., Park, C.-H., Son, Y.-W., Cohen, M. L. \& Louie, S. G. Quasiparticle energies and band gaps in graphene nanoribbons. Phys. Rev. Lett. 99, 186801 (2007).

22. Barone, V., Hod, O. \& Scuseria, G. E. Electronic structure and stability of semiconducting graphene nanoribbons. Nano Lett. 6, 2748-2754 (2006).

23. Son, Y.-W., Cohen, M. L. \& Louie, S. G. Energy gaps in graphene nanoribbons. Phys. Rev. Lett. 97, 216803 (2006).

24. Son, Y.-W., Cohen, M. L. \& Louie, S. G. Half-metallic graphene nanoribbons. Nature 444, 347-349 (2006).

25. Nakada, K., Fujita, M., Dresselhaus, G. \& Dresselhaus, M. S. Edge state in graphene ribbons: nanometer size effect and edge shape dependence. Phys. Rev. B 54, 17954-17961 (1996)

26. Niimi, Y. et al. Scanning tunneling microscopy and spectroscopy of the electronic local density of states of graphite surfaces near monoatomic step-edges. Phys. Rev. B 73, 085421 (2006).

27. Kobayashi, Y., Fukui, K., Enoki, T., Kusakabe, K. \& Kaburagi, Y. Observation of zigzag and armchair edges of graphite using scanning tunneling microscopy and spectroscopy. Phys. Rev. B 71, 193406 (2005).

28. Koskinen, P., Malola, S. \& Häkkinen, H. Evidence for graphene edges beyond zigzag and armchair. Phys. Rev. B 80, 073401 (2009).

29. Seitsonen, A. P. Structure and stability of graphene nanoribbons in oxygen, carbon dioxide, water, and ammonia. Phys. Rev. B 82, 115425 (2010).

30. Kroes, J. M. H., Akhukov, M. A., Los, J. H., Pineau, N. \& Fasolino, A. Mechanism and free-energy barrier of the type-57 reconstruction of the zigzag edge of graphene. Phys. Rev. B 83, 165411 (2011).

31. Wassmann, T., Seitsonen, A. P., Saitta, A. M., Lazzeri, M. \& Mauri, F. Structure, stability, edge states, and aromaticity of graphene ribbons. Phys. Rev. Lett. 101, 096402 (2008).

32. Kotakoski, J., Santos-Cottin, D. \& Krasheninnikov, A. V. Stability of graphene edges under electron beam: equilibrium energetics versus dynamic effects. ACS Nano 6, 671-676 (2012).

33. Kim, K. et al. Atomically perfect torn graphene edges and their reversible reconstruction. Nat. Commun. 4, 2723 (2013).

34. Li, X.-F., Wang, L.-L., Chen, K.-Q. \& Luo, Y. Electronic transport through zigzag/armchair graphene nanoribbon heterojunctions. J. Phys. Condens. Matter 24, 095801 (2012).

35. Li, T. C. \& Lu, S.-P. Quantum conductance of graphene nanoribbons with edge defects. Phys. Rev. B 77, 085408 (2008).

36. Sutter, P. \& Sutter, E. Microscopy of graphene growth, processing, and properties. Adv. Mater. 23, 2617-2634 (2013).

37. Man, K. L. \& Altman, M. S. Low energy electron microscopy and photoemission electron microscopy investigation of graphene. J. Phys. Condens. Matter 24, 314209 (2012).
38. Ishigami, M., Chen, J. H., Cullen, W. G., Fuhrer, M. S. \& Williams, E. D. Atomic structure of graphene on $\mathrm{SiO}_{2}$. Nano Lett. 7, 1643-1648 (2007).

39. Coraux, J., N'Diaye, A. T., Busse, C. \& Michely, T. Structural coherency of graphene on $\operatorname{Ir}(111)$. Nano Lett. 8, 565-570 (2008).

40. Meyer, J. C. et al. The structure of suspended graphene sheets. Nature 446, 60-63 (2007).

41. Girit, Ç. Ö. et al. Graphene at the edge: stability and dynamics. Science 323, 1705-1708 (2009).

42. Meyer, J. C. et al. Direct imaging of lattice atoms and topological defects in graphene membranes. Nano Lett. 8, 3582-3586 (2008).

43. Gass, M. H. et al. Free-standing graphene at atomic resolution. Nat. Nanotechnol. 3, 676-681 (2008).

44. Liu, Z., Suenaga, K., Harris, P. J. F. \& Iijima, S. Open and closed edges of graphene layers. Phys. Rev. Lett. 102, 015501 (2009).

45. Warner, J. H. et al. Structural transformations in graphene studied with high spatial and temporal resolution. Nat. Nanotechnol. 4, 500-504 (2009).

46. Xie, L. et al. Graphene nanoribbons from unzipped carbon nanotubes: atomic structures, Raman spectroscopy and electrical properties. J. Am. Chem. Soc. 113, 10394-10397 (2011).

47. Kotakoski, J., Krasheninnikov, A. V., Kaiser, U. \& Meyer, J. C. From point defects in graphene to two-dimensional amorphous carbon. Phys. Rev. Lett. 106, 105505 (2011).

48. Song, B. et al. Atomic-scale electron-beam sculpting of near-defect-free graphene nanostructures. Nano Lett. 11, 2247-2250 (2011).

49. Jin, C., Lan, H., Peng, L., Suenaga, K. \& Iijima, S. Deriving carbon atomic chains from graphene. Phys. Rev. Lett. 102, 205501 (2009).

50. Chuvilin, A., Meyer, J. C., Algara-Siller, G. \& Kaiser, U. From graphene constrictions to single carbon chains. New J. Phys. 11, 083019 (2009).

51. Dimiev, A. et al. Layer-by-layer removal of graphene for device patterning. Science 331, 1168-1172 (2011).

52. Zan, R., Ramasse, Q. M., Bangert, U. \& Novoselov, K. S. Graphene reknits its holes. Nano Lett. 12, 3936-3940 (2012).

53. Lu, C.-C. et al. Twisting bilayer graphene superlattices. Nano Lett. 7, 2587-2594 (2013).

54. Li, X., Cai, W., Colombo, L. \& Ruoff, R. S. Evolution of graphene growth on Ni and $\mathrm{Cu}$ by carbon isotope labeling. Nano Lett. 9, 4268-4272 (2009).

55 . Li, X. et al. Large-area graphene single crystals grown by low-pressure chemical vapor deposition of methane on copper. J. Am. Chem. Soc. 133, 2816-2819 (2011).

\section{Acknowledgements}

This work is supported by Grant-in-Aid for Scientific Research on Innovative Areas (MEXT KAKENHI Grant Number 25107003). Z.L., Y.-C.L. and K.S. acknowledge support from a JST Research Acceleration Program. P.-W.C. acknowledges project support from Taiwan Ministry of Science and Technology (NSC 100-2112-M-007-014-MY3).

\section{Author contributions}

C.-C.L., C.-H.Y. and P.-W.C. synthesized the materials. Z.L. and Y.-C.L. performed the experiments. Z.L. and Y.-C.L. analysed the data. Z.L., Y.-C.L., S.I. and K.S. co-wrote the paper. All authors discussed the results and commented on the manuscript.

\section{Additional information}

Supplementary Information accompanies this paper at http://www.nature.com/ naturecommunications

Competing financial interests: The authors declare no competing financial interests.

Reprints and permission information is available online at http://npg.nature.com/ reprintsandpermissions/

How to cite this article: Liu, Z. et al. In situ observation of step-edge in-plane growth of graphene in a STEM. Nat. Commun. 5:4055 doi: 10.1038/ncomms5055 (2014).

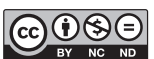

This work is licensed under a Creative Commons AttributionNonCommercial-NoDerivs 3.0 Unported License. The images or other third party material in this article are included in the article's Creative Commons license, unless indicated otherwise in the credit line; if the material is not included under the Creative Commons license, users will need to obtain permission from the license holder to reproduce the material. To view a copy of this license, visit http://creativecommons. org/licenses/by-nc-nd/3.0/ 